\title{
Alan Aderem
}

\begin{abstract}
Alan Aderem has long been working towards uniting two fields: infectious disease and systems biology. With his recent appointment as Director of Seattle BioMed - a 35-year-old global health institute in Washington, USA - he hopes to bring the two fields closer still. For this co-founder of the Seattle-based Institute for Systems Biology (ISB), the move marks an opportunity to fully integrate a systems approach into ongoing translational work on malaria, tuberculosis, HIV/AIDS and other infectious diseases. Asher Mullard sat down with Aderem to discuss his vision for Seattle BioMed.
\end{abstract}

\section{Q What drew you to infectious disease and systems biology? \\ I was practically born into infectious disease research, because my mother was a physician in a large black practice in South Africa and I effectively grew up in a township accompanying her. Then, when I was 23 , I was put under house arrest for 5 years because of my involvement in an anti-apartheid movement. I had nothing else to do during that time, so I did a Ph.D. in biophysics. When I later left the country, I realized that I wanted to put developing-world medicine together with physics. From there, I ended up working under Zanvil Cohn, studying innate immunity at Rockefeller University in New York, USA. \\ In the early 1990s, while the human genome was being sequenced, I started thinking a lot about what to do with all the data we'd be generating and developed the ideas that would turn out to be 'systems biology' When I met Lee Hood at a meeting, we realized that we had very similar ideas; we spoke through the night. Lee, Ruedi Aebersold and I eventually decided that we had tonnes of grants and lots of resources, so we should just start an Institute for the new field, which we did. We founded the ISB in 2000 and were practically the only place doing systems biology.}

\section{Q Why have you now chosen to move to Seattle BioMed?}

Initially, I used the immune system as a test bed to develop systems biology tools.

I couldn't really turn its force onto infectious disease because we had to develop the new approaches first. But I was always thinking about what a fantastic tool systems biology would be for translation. Since the foundation of the ISB, however, the field has developed; I can't think of a major university that doesn't now have a systems biology initiative. I feel that we now know enough to start applying our systems approaches to infectious diseases, in the context of vaccines and drugs.

It turned out that Ken Stuart, who founded Seattle BioMed in 1976, had been thinking in the opposite direction, about doing global health in the context of systems biology. One thing that's fantastic about Seattle BioMed is that all of the key infectious diseases are represented in its research programme, as is immunology. In addition, it has a unique malaria clinical trial centre where we can do experimental work. Ken and I realized that Seattle BioMed would be a great place to combine infectious disease and systems biology.

\section{Is infectious disease particularly tractable} to a systems biology approach?

Yes, I think it is. In part, this is because the cells of the immune system are accessible. Blood is a window into the disease; without invasion, we can get blood cells out from patients, isolate immune cells and examine these in all the ways we need to. I think the US National Institutes of Health is very aware of the power of the systems approach for infectious disease research as well, and is funding it and engaging with the people who are doing the analysis.

\section{Q What do you see systems biology bringing to the clinic?}

I would say that the first things that are going to happen are biomarkers. This is going to be the case with cancer - which has been at the forefront of systems biology analysis - and also with vaccine development. Correlates of immunity are going to be very useful for establishing early on whether or not a vaccine is going to work.
In regard to vaccines, however, I also think that systems biology will provide us with important basic-biology understanding of disease and immunity. With HIV/AIDS and tuberculosis, for example, we still need to figure out what kinds of immune responses are needed to fight infection, because we don't naturally develop defences against these. I think systems approaches can be helpful here, because rather than look at a single protein here or response there, we can look at the system in its totality.

\section{Q How fully is industry embracing systems approaches?}

I don't think that industry will ever do full systems analyses themselves. Companies need to answer to shareholders who want to know what has been done for profitability each quarter, whereas developing systems biology approaches and harnessing their potential for drug discovery needs a long-term view. Also, systems biology requires collaboration between a lot of different types of people; here at Seattle BioMed, we are establishing a team that will include pure and applied mathematicians, physicist and astrophysicists working on projects. These are not the usual scientists who interact with biologists in a company.

But that said, we can't take a product to the stage at which it is a vial on a shelf in a pharmacy in Africa on our own. So we need to partner with industry. In August 2011, for instance, GlaxoSmithKline and Crucell are beginning a big trial for the prime-boost RTS,S anti-malarial vaccine, and we are doing the systems biology for this trial. This is the first vaccine trial in which a systems approach has been built into the protocol, and I think it will be an interesting model. 